\title{
Electron accelerating unit for streak image tubes
}

\author{
FANGKE ZONG, QINLAO YANG*, HOUZHI CAI, LI GU, \\ XIANG LI and JINGJIN ZHANG
}

Key Laboratory of Optoelectronic Devices and Systems of the Ministry of Education and Guangdong Province, College of Optoelectronic Engineering,

Shenzhen University, Shenzhen 518060, China

e-mail: qlyang@szu.edu.cn

MS received 18 November 2014; revised 18 June 2015; accepted 17 September 2015

\begin{abstract}
An electron accelerating unit is proposed for use in streak image tubes (SITs). An SIT with this new accelerating unit was simulated using the Monte Carlo method. The simulation results show that the accelerating unit improves both the spatial and temporal resolution. Compared to a traditional SIT, the transit time spread for electrons in the cathode-to-mesh region is reduced from 247 to $162 \mathrm{fs}$, the line width of the electron beam on the image surface is reduced from 42.7 to $26.1 \mu \mathrm{m}$, and the temporal resolution is improved from 515 to $395 \mathrm{fs}$.
\end{abstract}

Keywords. High-speed photography; streak camera; Monte Carlo; electro-optical devices.

\section{Introduction}

Streak cameras are among the most valuable instruments for recording ultrafast phenomena in laser-driven inertial confinement fusion (ICF) experiments, and streak image tubes (SITs) are a core part of streak cameras. Fast ignition techniques proposed for ICF experiments require SITs with much higher temporal resolution. Since the first sub-picosecond SIT temporal resolution was reported by Sibbett et al (1982), there has been significant progress in the fundamental theory and manufacturing technology for SITs. Many new methods and technologies have been developed for the design of femtosecond SITs. Shakya \& Chang (2005) reported that the temporal resolution of the camera reached $280 \mathrm{fs}$ and it was done by changing the electron beam size in the deflection plates with a variable slit in front of the plates. Hares \& Dymoke-Bradshaw (2008) describes a new instrument in which the extraction electrostatic field at the photocathode increases with time, converting time to PE energy. A uniform magnetic field is used to measure the PE energy, and thus time, and also focuses in one dimension. Design calculations are presented for the factors limiting the time resolution. Feng et al (2010) realized an ultrafast X-ray streak camera with $600 \mathrm{fs}$ temporal resolution by using a grazing incidence reflection photocathode. However, current SITs are still not sufficient for the fast ignition technique.

*For correspondence 
In the present study we calculated the transit time spread (TTS) of electrons in various regions and propose a new electron accelerating unit to reduce the TTS. TTS in the cathodeto-mesh region mainly arises from different emission angles and transmission paths, which can be reduced by a self-focusing field generated from the accelerating unit. Moreover, application of a pulsed high voltage to the accelerating electrode enhanced the field strength near the photocathode surface and reduced the background noise produced by stray and hot electrons. This improves the signal to noise ratio (SNR).

An SIT with the new accelerating unit was designed and simulated based on the Monte Carlo method. Compared to a traditional SIT, the electron TTS is reduced from 247 to $162 \mathrm{fs}$ in the cathode-to-mesh region, the temporal SIT resolution is increased by $23.3 \%$, and the line width of the electron beam on the image surface is reduced from 42.7 to $26.1 \mu \mathrm{m}$. These simulation results demonstrate that the proposed accelerating unit can improve both the spatial resolution and the temporal resolution.

\section{Accelerating unit design}

The temporal resolution of femtosecond SITs is limited by the initial energy distribution, the space charge effect, and the TTS of photoelectrons emitted from the photocathode. The initial energy distribution is related to the photocathode materials. Niu \& Sibbett (1981) reported that the space charge effect depends on the transit time of photoelectrons and the geometry of the electron beam during transmission. Factors that influence the TTS are the initial energy spread, the transit time and the space charge effect of photoelectrons emitted from the photocathode. Thus, choosing a photocathode with a small initial photoelectron energy distribution and enhancing the field are common methods for reducing the TTS, this is in accordance with Kinoshita et al (1987).

Bai et al (2011) designed a femtosecond soft X-ray SIT with a large dynamic range. The authors reported that temporal resolution of $500 \mathrm{fs}$ had been obtained. Our proposed electron accelerating unit was introduced into this SIT, for which the geometry is shown in figure 1 . The accelerating unit comprises an accelerating electrode with a thickness of $0.1 \mathrm{~mm}$ and a slit width of $0.1 \mathrm{~mm}$ between the cathode and the mesh. The distance between the cathode and the mesh is $1.2 \mathrm{~mm}$. The electric field in the cathode-to-mesh region is a self-focusing field, which can reduce the electron beam size.

There are three advantages of the accelerating unit. In the pulsed mode, a pulsed voltage is applied to the accelerating electrode. First, the SIT works only when a signal is detected, which reduces the background noise produced by stray and hot electrons under non-working

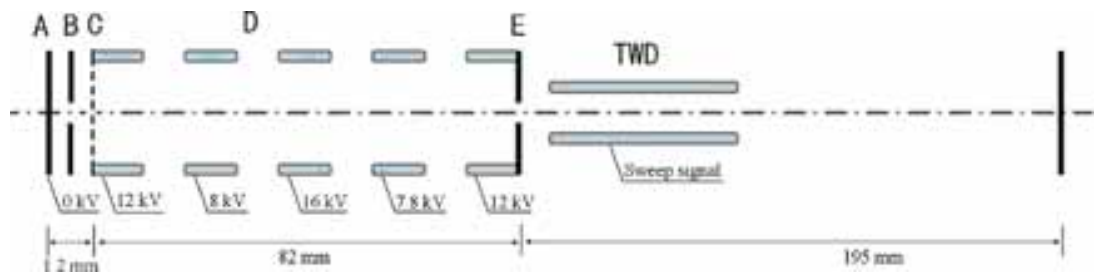

Figure 1. Geometry of the SIT with the new accelerating unit: (A) photocathode; (B) accelerating electrode; (C) mesh; (D) focus electrodes; (E) anode; and TWD, traveling wave deflector. The total length of the SIT is $278.2 \mathrm{~mm}$, the focusing system length is $82 \mathrm{~mm}$. The voltage applied to each electrode is 12,8 , $16,7.8$, and $12 \mathrm{kV}$, respectively. Sweep signal is applied to the TWD. 
conditions, thereby improving the image quality. Second, a much higher acceleration field can be obtained by a pulsed compared to a static voltage which was identified by Degtyareva et al (2003). The field strength near the photocathode surface is significantly enhanced, which improves the temporal resolution. Third, a pulsed voltage is applied to the accelerating electrode instead of the photocathode, so it is easier to obtain a consistent and steady field near the cathode surface. These factors favor improvements in the temporal resolution.

\section{Results and analysis}

In traditional SITs, the cathode and the mesh are parallel to each other. When the electron beam traverses the planar electric field between them, a time spread is generated. The time spread can be described by an empirical equation proposed by Schelev et al (1971). The electron TTS in the cathode-to-mesh region can be estimated as

$$
\Delta t_{\mathrm{cm}}=2.34 \times \frac{\sqrt{\delta \varepsilon}}{E_{\mathrm{c}}}(\mathrm{ps}),
$$

where $\delta \varepsilon$ is the full width at half-maximum of the initial photoelectron energy distribution [eV] and $E_{\mathrm{c}}$ is the extraction field strength $[\mathrm{kV} / \mathrm{mm}]$.

When the electron beam traverses the field between the deflection plates, the top and bottom parts of the beam will be at different potentials in the transverse section, so a time dispersion exists. Jaanimagi (2004) proposed an empirical equation to describe this time dispersion and it can be approximated as

$$
\Delta t_{\mathrm{d}}=\frac{2 \alpha \omega}{v_{\mathrm{a}}},
$$

where $\alpha[\mathrm{rad}]$ is the deflection angle of the electron beam when it traverses the deflection plates, $v_{\mathrm{a}}$ is the average electron axial velocity, and $2 \omega$ is the transverse size of the electron beam at the entrance to the deflection plates. Thus, $\Delta t_{\mathrm{d}}$ is directly proportional to the electron beam size $2 \omega$.

When the photocathode is irradiated by a light pulse, a great number of electrons are excited from the photocathode surface. This electron emission process is simulated as a statistical sample in terms of Monte Carlo Method in proper probability. The quantity and location of the electrons are sampled randomly in proportion to the light intensity distribution. The initial energy, emission angle, and launch time are sampled randomly from beta, Lambertian, and Gaussian probability distributions, respectively. In the electron optics focusing system, the electron motion is dependent on the electric field force. The nonrelativistic electron motion equation in the electrostatic field can be written as

$$
\frac{d v(t)}{d t}=\frac{e}{m_{e}} \frac{\partial \varphi(r)}{\partial r}
$$

where $m_{e}$ is the electron mass, $v(t)$ is the electron velocity, $\varphi(r)$ is the potential distribution. The electrostatic field is determined by Laplace's equation, which can be solved by using the finite difference method. The electron motion equation is a second order differential equation, which can be solved by using fourth order Runge-Kutta method. In simulation, we wrote our own solver using above method in MATLAB language. First the potential distribution is obtained by 
solving Laplace's equation, and then the trajectory and velocity of each electron are calculated by solving the electron motion equation.

Presumed that a slit of $1 \mathrm{~mm}$ long and $0.01 \mathrm{~mm}$ wide on the photocathode is irradiated by a light pulse of $50 \mathrm{fs}$ duration, if 500 photoelectrons are excited from this area, then the initial current density will be $8 \mathrm{~A} / \mathrm{cm}^{2}$. By tracing these electrons trajectories and analyzing the flight time distribution, the temporal characters of the SIT will be obtained. For a traditional SIT, $\Delta t_{\mathrm{cm}}=247 \mathrm{fs}$ and $\Delta t_{\mathrm{ca}}=476 \mathrm{fs}$. In the new SIT, $\Delta t_{\mathrm{cm}}=216 \mathrm{fs}$ and $\Delta t_{\mathrm{ca}}=442 \mathrm{fs}$ were obtained for an accelerating voltage of $6 \mathrm{kV}$. When the extracting field strength $E_{\mathrm{c}}$ was enhanced to $14.5 \mathrm{kV} / \mathrm{mm}, \Delta t_{\mathrm{cm}}=162 \mathrm{fs}$ and $\Delta t_{\mathrm{ca}}=361 \mathrm{fs}$ were obtained. Compared to the traditional SIT, TTS decreased by $24 \%$ in the cathode-to-anode region.

The line width of the electron beam on the image surface was calculated as 48.1, 42.2, 37.2 and $26.1 \mu \mathrm{m}$ for slit electrode voltage of $5,6,7$, and $8 \mathrm{kV}$, respectively. The line width for the traditional SIT was $42.7 \mu \mathrm{m}$. Thus, thespatial resolution improved with increasing $E_{\mathrm{c}}$.

Figure 2 shows that TTS in the cathode-to-mesh and cathode-to-anode regions decreased with increasing accelerating voltage. These results are consistent with Degtyareva et al (2005), who proposed that an increase in electric field strength near the cathode surface can improve the temporal resolution.

The spatial extent of the electron beam was calculated for various voltages applied to the accelerating electrode, as shown in figure 3 . The beam size in transverse profile at the entrance to the deflection plates was $0.51,0.79,0.94$, and $1.12 \mathrm{~mm}$ for voltage of $5,6,7$, and $8 \mathrm{kV}$, respectively. The beam size was $0.87 \mathrm{~mm}$ in the traditional SIT. These results demonstrate that the electron beam broadened with increasing voltage at the accelerating electrode. This is inconsistent with the improvement in temporal resolution with increasing voltage. Therefore, introduction of an aperture was required to solve this problem. An aperture with a slit was placed at the entrance to the deflection plates to restrict the electron beam size. Figure 4 shows the electron transmission through the aperture for different slit widths. Considering that the electron interception rate affects the SNR of the SIT, a slit width of $0.7 \mathrm{~mm}$ was chosen. For this aperture, $3 \%$, 5\%, and $11 \%$ of the electrons were intercepted by the aperture for accelerating electrode voltages of 6,7 , and $8 \mathrm{kV}$, respectively.

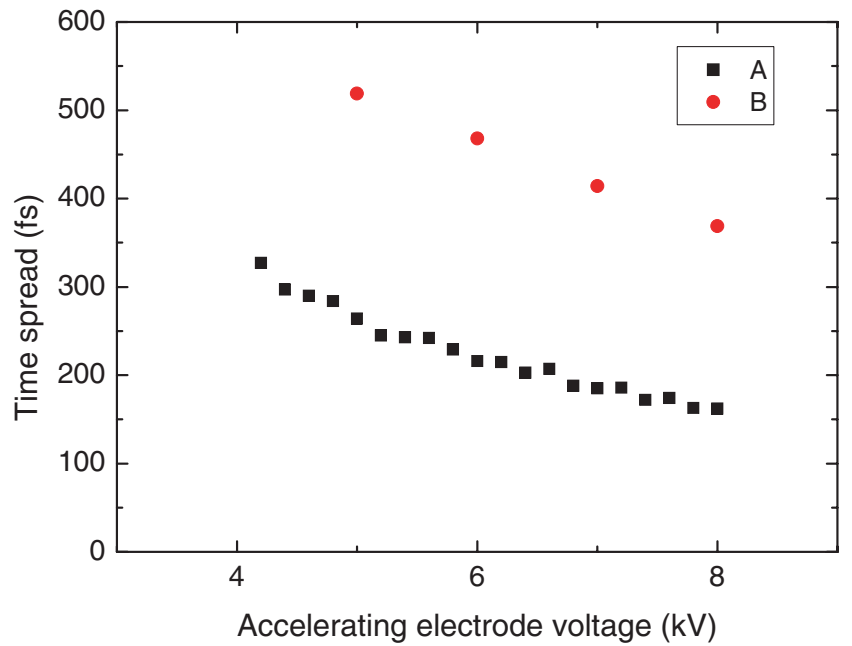

Figure 2. TTS in (A) the cathode-to-mesh region and (B) the cathode-to-anode region. 

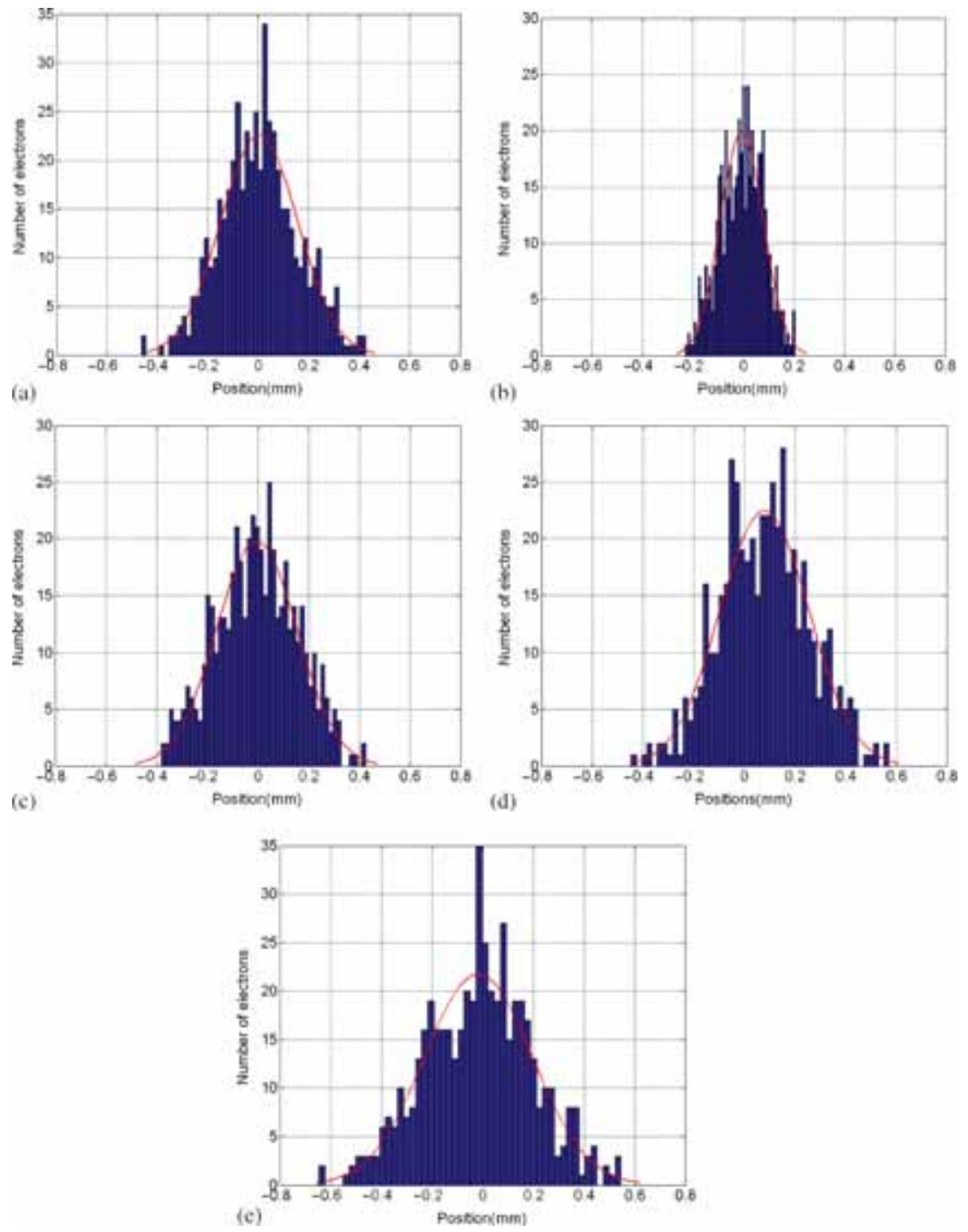

Figure 3. Transverse profiles of the SIT electron beam for (a) a traditional SIT without an accelerating unit and an SIT with the proposed accelerating unit at voltage of (b) 5, (c) 6, (d) 7, and (e) $8 \mathrm{kV}$.

In SIT simulations, values of $\delta \varepsilon=1.1 \mathrm{eV}$ obtained by Henke et al (1979), $E_{c}=10 \mathrm{kV} / \mathrm{mm}$, $\alpha=0.015 \mathrm{rad}$, and $\nu_{\mathrm{a}}=6.5 \times 10^{7} \mathrm{~m} / \mathrm{s}$ were used. The results calculated are listed in table $1 . V_{\mathrm{s}}$ is the voltage applied to the accelerating electrode, $\Delta t_{\mathrm{ca}}$ is the electron TTS in the cathode-to-anode region, and $\Delta t$ is the temporal SIT resolution, which can be estimated as $\Delta t=\sqrt{\Delta t_{\mathrm{ca}}^{2}+\Delta t_{\mathrm{d}}^{2}}$. Temporal resolution of 515 fs was obtained for the traditional SIT.

The temporal resolution of the SIT was calculated for various voltages applied to the accelerating electrode, as shown in figure 5. The results show that the temporal resolution of the tube improved from 532 to 395 fs when $V_{\mathrm{s}}$ was increased from 5 to $8 \mathrm{kV}$. Compared to the traditional tube, the temporal resolution was improved by $23.3 \%$. 


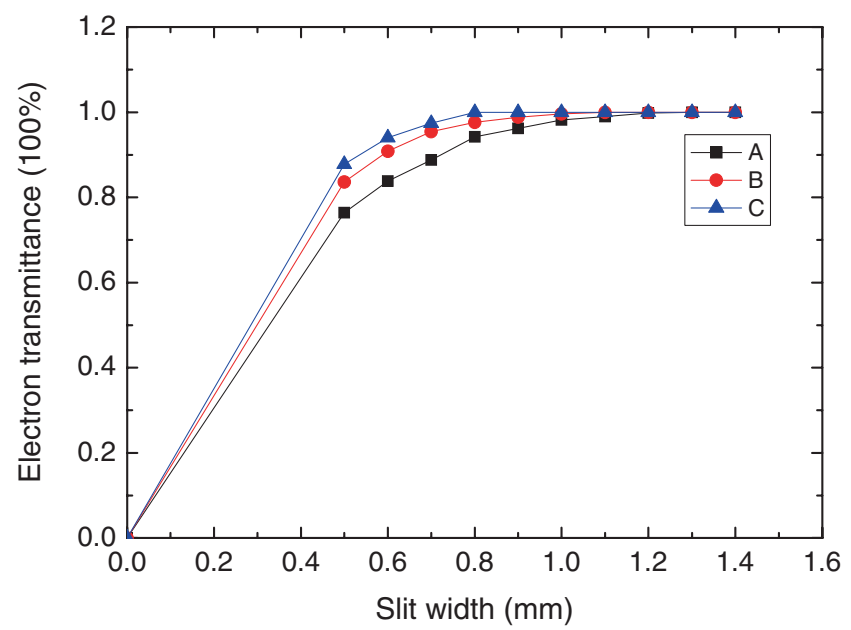

Figure 4. Electron transmittance through the aperture as a function of slit width for accelerating voltage of (A) 8, (B) 7, and (C) $6 \mathrm{kV}$.

Table 1. TTS results calculated for the streak image tube.

\begin{tabular}{lccc}
\hline$V_{\mathrm{s}}(\mathrm{kV})$ & $\Delta t_{\mathrm{d}}(\mathrm{fs})$ & $\Delta t_{\mathrm{ca}}(\mathrm{fs})$ & $\Delta t(\mathrm{fs})$ \\
\hline 5 & 117 & 519 & 532 \\
6 & 159 & 469 & 495 \\
7 & 159 & 419 & 448 \\
8 & 159 & 362 & 395 \\
\hline
\end{tabular}

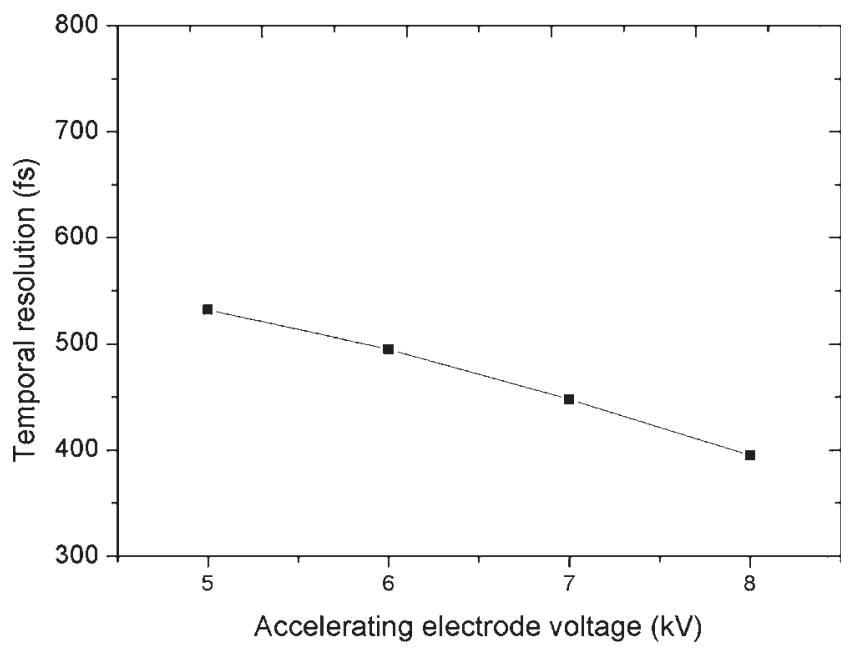

Figure 5. Theoretic results of the temporal resolution of the SIT. 


\section{Conclusions}

An electron accelerating unit for SITs was proposed. The spatial and temporal SIT resolution were calculated according to the Monte Carlo method. The electron beam size at different positions was calculated. Compared to a traditional tube, the temporal SIT resolution was improved by $23.3 \%$ and the line width of the electron beam on the image surface was reduced from 42.7 to $26.1 \mu \mathrm{m}$. The results demonstrate that the proposed electron acceleration unit improves the spatial and temporal SIT resolution.

\section{Acknowledgement}

This study is supported by the National Natural Science Foundation of China (NSFC) under Grant No. 11305107, and the National Science and Technology Program of Shenzhen under Grant No. JCYJ20140418182819143.

\section{References}

Bai X, Niu H, Yang Q, Li J, Liao H and Niu L 2011 Large dynamic range femtosecond soft X-ray streak image tube. J. High Power Laser Particle Beams 23(10): 2683-2686

Degtyareva V P, Belolipetski V S, Bryukhnevich G I, Ivanova S R, Levina G P, Makushina V A, Polikarkina N D, Semichastnova Z M and Schelev M Y 2003 Femtosecond streak tubes designing, manufacturing and testing. In: Proceedings of 25th International Congress on High-Speed Photography and Photonics SPIE, 4948: 281-290

Degtyareva V P, Bryukhnevich G I, Lozovoi V I, Schelev M Y, Semichastnova Z M and Vorobiev N S 2005 Dynamic parameters evaluation for femtosecond streak tubes. In: Proceedings of 26th International Congress on High-Speed Photography and Photonics, SPIE, 5580: 416-423

Feng J, Engelhorn K, Cho B I, Lee H J, Greaves M, Weber C P, Falcone R W, Padmore H A and Heimann P A 2010 A grazing incidence X-ray streak camera for ultrafast, single-shot measurements. J. Appl. Phys. Lett. 96(13): 134102

Henke B L, Liesegang J and Smith S D 1979 Soft-X-ray-induced secondary-electron emission from semiconductors and insulators: Models and measurements. J. Phys. Rev. B 19(6): 3004-3021

Hares J D and Dymoke-Bradshaw A K L 2008 A novel compact high speed X-ray streak camera (invited). J. Rev. Sci. Instrum. 79(10): 10F502

Jaanimagi P A 2004 Breaking the 100-fs barrier with a streak camera. In: Proceedings of Fourth-Generation X-Ray Sources and Ultrafast X-Ray Detectors, SPIE, 5194: 171-184

Kinoshita K, Ito M and Suzuki Y 1987 Femtosecond streak tube. J. Rev. Sci. Instrum. 58(6): 932-938

Niu H and Sibbett W 1981 Theoretical analysis of space-charge effects in photochron streak cameras. J. Rev. Sci. Instrum. 52(12): 1830-1836

Shakya M M and Chang Z 2005 Achieving 280 fs resolution with a streak camera by reducing the deflection. J. Appl. Phys. Lett. 87: 041103

Schelev M Y, Richardson M C and Alcock A J 1971 ImageConverter streak camera with picosecond resolution. J. Appl. Phys. Lett. 18(8): 354-357

Sibbett W, Niu H and Baggs M R 1982 Photochron IV subpicosecond streak image tube. J. Rev. Sci. Instrum. 53(6): 758-761 